\title{
Programa Estudiantes Mentores, "Una expe- riencia valiosa en la FAREM-Carazo"
}

MSc. Juanita Rodríguez Lara Máster en Administración y Gestión de la Educación UNAN-MANAGUA, FAREM-CARAZO jrodrilar@yahoo.com

MSc. Pedro Silvio Conrado González Máster en Didácticas Específicas con

Administración del Currículo UNAN-MANAGUA, FAREM-CARAZO pedrosilvioc081180@hotmail.com

Palabras Clave: Estudiante mentor, mentoría, problemas de aprendizaje, éxito académico, seguimiento y evaluación.

\section{RESUMEN}

E I programa de alumnos mentores se inició en el 2016, en el primer semestre con estudiantes de primer ingreso y con las asignaturas básicas como son: Lengua y Literatura, Introducción a la Física, Introducción a la Química y Matemática General, como iniciativa de la dirección de docencia de grado en conjunto con los departamentos docentes de la UNAN-Managua.

Uno de los problemas más comunes con los que se encuentra un alumno de primer ingreso en el entorno universitario es su profundo desconocimiento del mismo, sobre todo si tenemos en cuenta el cambio radical en cuanto a metodología de trabajo y estudio con el que se afronta, lo que dificulta aún más su integración en la vida universitaria.

Una de las medidas que se toman en UNAN-Managua, específicamente en la Facultad Regional de Carazo, es la implementación del programa alumnos mentores, quienes con su disponibilidad han permitido que el proyecto en este primer semestre del 2016 haya sido todo un éxito mejorando la calidad en el estudiantado y por ende, el rendimiento académico en la facultad.

En este artículo se describe en detalle la experiencia llevada a cabo en la FAREM-Carazo, sobre la puesta en marcha del programa de mentoría. En el Proyecto Mentor los estudiantes de más de un año académico y bajo la supervisión de los profesores, ayudan y orientan a los estudiantes con algunos problemas académicos para que puedan superarlo y la experiencia muestra que se ha logrado el objetivo de mejorar la calidad y el rendimiento académico.

Cabe recalcar, que el Departamento de Ciencias de la Educación y Humanidades fue el que estuvo al frente de este programa ejecutado por el equipo coordinador y dirigido por la Unidad Metodológica. La mayoría de los mentores fueron los alumnos del mismo departamento quienes 
se están formando para ser docentes, con excepción de dos estudiantes del Departamento de Ciencia Tecnología y Salud quienes asumieron el reto y lo cumplieron a cabalidad (un alumno de segundo año de Ingeniería Industrial que trabajó con la asignatura Introducción a la física y una alumna de tercer año de Bioanálisis Clínico que trabajó con Introducción a la Química).

\section{INTRODUCCIÓN}

La Universidad Nacional Autónoma de Nicaragua, a través del Vicerrectorado de Docencia, impulsa el "Programa de Estudiantes Mentores", encuentra su fundamento en el Modelo Educativo, Normativa y Metodología para la Planificación Curricular, específicamente con su Fundamentación Epistemológica (p.25). Esta expresa que, "no todas las personas aprendemos al mismo ritmo", "el conocimiento tiene un carácter constructivista social e interactivo" y, finalmente, que "el conocimiento no se transmite, sino que se construye en la práctica".

En este mismo sentido, el Modelo Pedagógico (p.31) expresa que, el proceso de enseñanzaaprendizaje se centra en el estudiante y en el desarrollo de procedimientos, habilidades, estrategias y técnicas que les permitan aprender a aprender [...]

A través del Programa se dio cobertura y atención estudiantil y por ende, la mejora en la calidad de los aprendizajes. Este fue ejecutado por el equipo coordinador en conjunto con la Unidad Metodológica que se encarga de brindar herramientas en metodologías didácticas, estrategias de enseñanza y planeación didáctica, proceso de capacitación que permitió a los estudiantes mentores ponerse al frente de sus compañeros de universidad en el reforzamiento de los contenidos de mayor dificultad.

Las asignaturas objeto de seguimiento para el primer semestre 2016, fueron: Lengua y Literatura, Introducción a la Física, Introducción a la Química y Matemática General, los estudiantes mentores fueron seleccionados por los coordinadores de carrera, teniendo como criterios de selección más importantes: la excelencia académica, disponibilidad para el programa de mentores, liderazgo y actitud positiva hacia la docencia.

\section{OBJETIVOS DEL PROGRAMA EN LA FAREM-CARAZO}

a. Promover el programa de estudiantes mentores a través de los docentes que imparten las asignaturas objeto de seguimiento y contempladas en el programa.

b. Capacitar a estudiantes mentores en estrategias didácticas, planificación y control de grupo para un buen desempeño en el desarrollo de las mentorías.

c. Facilitar material didáctico a los estudiantes mentores que permitan el buen desarrollo de la actividad didáctica.

d. Dar seguimiento a estudiantes mentores en el desarrollo de sus actividades didácticas con sus mentorizados.

e. Ayudar a los estudiantes mentorizados para el éxito en las asignaturas con problemas de aprendizaje. 


\section{MARCO TEÓRICO}

En este apartado se describe el fundamento teórico de este trabajo, se inicia con la del alumno mentor y su importancia en el desarrollo de competencias del estudiantado universitario novel, y termina con las diferencias entre alumno mentor y alumno ayudante y sus utilidades en el desarrollo de competencias en el estudiantado.

\subsection{Alumno mentor y su importancia en el desarrollo de competencias del estudiantado universitario novel}

La Universidad, en su función social y académica, busca la formación de profesionales integrales capaces de hacer aportes oportunos a la realidad en la que se encuentra, en gran medida, la calidad y eficiencia de la educación depende del nivel de preparación de sus docentes, calidad que se adquiere en la formación profesional y en el ejercicio mismo de la docencia, pero al mismo tiempo, de la dedicación y motivación de los estudiantes hacia el aprendizaje. Los docentes deben ser capaces de colaborar en el proceso de adaptación de los alumnos noveles al entorno universitario, para evitar el abandono y el fracaso estudiantil.

La UNAN-Managua, consciente de esta función social en su Modelo Educativo, establece como función "formar profesionales y técnicos integrales desde y con una concepción científica y humanista del mundo capaces de interpretar los fenómenos sociales y naturales con un sentido crítico, reflexivo y propositivo para que contribuyan al desarrollo social" (UNAN-Managua, 2011, pág. 19).

Sin embargo, los docentes encuentran alumnos que viven en un entorno diferente, que conciben al mundo de otra manera y que han nacido en un entorno digital, en donde los avances de la tecnología son parte de su vida y que los incorporan a las aulas de clase. No es ajeno observar celulares de última generación, Tablet, y acceso a la Internet como algo común, en ese sentido, se debe estar consciente de que los universitarios de hoy son la primera generación de nativos digitales (Rodríguez 2015, pág. 100) y esta generación de alumnos tienen sus propias características que los identifican. Sigue diciendo el estudioso, que son, a la vez, los más conectados y los más solitarios y aislados que hayan pasado por las aulas.

Además de las funciones académicas, los docentes tienen la función de colaborar en la adaptación de los alumnos noveles al entorno universitario porque estos son ajenos a las formas de trabajo y a la cultura universitaria.

Los nuevos alumnos no conocen las formas de trabajo y la cultura universitaria y esto les dificulta su adaptación a la vida universitaria. La imagen y la idea que tienen de la Universidad la mayoría de ellos, responden fundamentalmente a estereotipos de un modelo de Universidad que no se corresponde con la actual realidad de esta Institución (Rodríguez, 2015, pág. 106).

Ante la situación planteada, surge la figura del alumno mentor, estudiantes de los últimos años o de años superiores, que con características particulares y vocación de servicio, colabora con los alumnos, con mayores dificultades de aprendizaje, porque este ya está adaptado al entorno universitario y al mismo tiempo, tiene dominio de su campo de estudio y solo debe ser preparado en el manejo de estrategias de aprendizaje.

Un mentor es alguien que ayuda a aprender algo que no se hubiese aprendido o que hubiese 
aprendido más lentamente o con mayor dificultad, de haberlo tenido que hacer por cuenta propia (Car, 1999). En este sentido, este alumno actúa como tutor o asesor de uno o varios estudiantes con problemas de asimilación de los aprendizajes en el área que más dominio tiene. La tutoría que el mentor brinda se centra principalmente en superar los problemas de aprendizaje de los estudiantes tutelados; por lo cual, el Estudiante Mentor debe tener excelente dominio de su campo de estudio y de igual manera, debe conocer estrategias de aprendizaje efectivas.

Como se observa claramente, la mentoría brinda la oportunidad de igualar a aquellos alumnos que por cualquier razón no hayan asimilado un contenido dado y potencia de esta manera su desarrollo intelectual y de aprendizaje. En ese mismo sentido, Ruiz de Miguel et al (2004, p. 92) definen mentoría, como un proceso de feed-back continúo de ayuda y orientación entre el mentor (alumno de curso superior que atesora los conocimientos y habilidades necesarias para ayudar) y un alumno o un grupo de alumnos de nuevo ingreso, con la finalidad de paliar las necesidades de estos y optimizar su desarrollo y potencial de aprendizaje.

Es importante no confundir la función de la mentoría con las funciones de los alumnos ayudantes - las ayudantías, que son estudiantes que asisten o colaboran al docente en sus funciones, ya sean de gestión, docencia, investigación o extensión académica.

\subsection{Diferencias entre el alumno mentor y los alumnos ayudante}

Es importante hacer notar la diferencia entre un alumno llamado alumno-ayudante y un estudiante mentor. Este último realiza su función sin ningún interés económico, solamente humanista, con el fin de colaborar con sus compañeros, y un alumno-ayudante en Nicaragua, va más allá del humanismo, pues recibe una ayuda económica; por tanto, un alumno mentor tiene mucho mérito ya que cumple con la misión de la Universidad de formar profesionales íntegros y humanistas que piensen en el bien común.

Sin embargo, tampoco hay que restarle mérito al programa de alumno-ayudante, pues constituye una poderosa herramienta de intervención que permite experimentar y mejorar las habilidades sociales a través de la propia práctica (Andrés y Barrios, 2006, pág. 315). Es decir, en sus funciones han sido de mucha ayuda en el quehacer universitario, aquí en FAREM-Carazo, ellos prestan colaboración en los laboratorios, labores de gestión, muy poco en la labor docente, función que fue desempeñada por los alumnos mentores.

\section{METOdOlOgÍA DE TRABAJO}

La metodología propuesta para el desarrollo del taller fue de carácter activo y participativo, basados en el enfoque de aprender-haciendo, en el que los participantes fueron los gestores de sus conocimientos y se apropiaron de los mismos con ayuda del docente facilitador.

Este taller se inició con una autoevaluación sobre estrategias didácticas, en el que los participantes reflexionaron sobre las estrategias didácticas que conocen y las que se podían aplicar con los estudiantes mentorizados.

En la segunda sesión, se tomaron aspectos de la forma de planificación que debían considerar para el proceso de mentorías; también caracterizaron a un buen mentor como una persona responsable, con carácter de líder y sobre todo, humanista con espíritu de servicio. 
En la evaluación se fue objetivo, apartando influencias subjetivas, los criterios fueron sencillos, basados en la participación, asistencia y aportes significativos.

\section{DESARROLLO}

El Programa se desarrolló durante el primer semestre del año 2016, en tres fases principales:

\section{Primera fase: Reunión con coordinadores de carrera}

El objetivo principal de esta fase fue la selección de los estudiantes mentores, por parte de los coordinadores de carrera de Lengua y Literatura, Ciencias Naturales y Física-Matemática.

\section{Segunda fase: Selección de los alumnos mentores y propuesta de mentoriados}

La selección de los alumnos mentores fue tomando en cuenta los criterios establecidos en el programa, que consistían en que fuesen alumnos destacados, con espíritu de servicio y vocación docente. Se presenta así la propuesta inicial de 31 alumnos. Ver Tabla 1.

Tabla 1. Propuesta inicial de alumnos mentores.

\begin{tabular}{|l|c|}
\hline \multicolumn{1}{|c|}{ ASIGNATURAS ATENDIDAS } & NO. DE ESTUDIANTES \\
\hline Química y Biología & 4 \\
\hline Física y Matemática & 17 \\
\hline Técnicas de Lectura, redacción y ortografía & 10 \\
\hline
\end{tabular}

Sin embargo, durante el desarrollo del programa solamente cumplieron con su asignación 17 estudiantes mentores.

\section{Tercera fase: Proceso de mentoría y seguimiento}

Las carreras atendidas pertenecían a los tres departamentos docentes de Ciencias de la Educación y Humanidades (Ciencias Naturales, Física- Matemática, Ciencias Sociales, Lengua y Literatura, Pedagogía y Psicología); Ciencias económicas y administrativas (Administración de Empresas y Mercadotecnia); Ciencias Tecnología y Salud (Ingeniería Industrial e Ingeniería en Sistemas), participaron un total de 119 estudiantes. Ver tabla 2.

Tabla 2. Informe estadístico alumnos mentores, I Semestre 2016.

\begin{tabular}{|c|c|c|c|c|c|c|c|c|c|}
\hline \multirow{2}{*}{$\begin{array}{l}\text { ASIGNATURA } \\
\text { ATENDIDA }\end{array}$} & \multirow[t]{2}{*}{ CARRERAS } & \multicolumn{4}{|c|}{ ALUMNO MENTORES } & \multicolumn{4}{|c|}{$\begin{array}{c}\text { ALUMNOS } \\
\text { MENTORIZADOS }\end{array}$} \\
\hline & & $\mathbf{V}$ & $\%$ & $\mathbf{M}$ & $\%$ & $\mathbf{V}$ & $\%$ & $M$ & $\%$ \\
\hline \multirow{3}{*}{$\begin{array}{l}\text { Introducción a } \\
\text { la Química }\end{array}$} & Ingeniería Industrial & - & - & 1 & - & 3 & 25 & 9 & 75 \\
\hline & Ciencias Naturales (G1) & - & - & 1 & - & 1 & 20 & 4 & 80 \\
\hline & Ciencias Naturales (G1) & 1 & - & - & - & 1 & 20 & 4 & 80 \\
\hline \multicolumn{2}{|r|}{ Sub total } & 1 & 33 & 2 & 67 & 5 & 23 & 17 & 77 \\
\hline \multirow{2}{*}{$\begin{array}{l}\text { Introducción a } \\
\text { la Física }\end{array}$} & Ciencias Naturales & 1 & - & - & - & 2 & 20 & 8 & 80 \\
\hline & Física Matemática & - & - & 1 & - & 2 & 20 & 8 & 80 \\
\hline \multicolumn{2}{|r|}{ Sub total } & 1 & 50 & 1 & 50 & 4 & 20 & 16 & 80 \\
\hline
\end{tabular}




\begin{tabular}{|c|c|c|c|c|c|c|c|c|c|}
\hline \multirow{2}{*}{$\begin{array}{l}\text { ASIGNATURA } \\
\text { ATENDIDA }\end{array}$} & \multirow[t]{2}{*}{ CARRERAS } & \multicolumn{4}{|c|}{ ALUMNO MENTORES } & \multicolumn{4}{|c|}{$\begin{array}{c}\text { ALUMNOS } \\
\text { MENTORIZADOS }\end{array}$} \\
\hline & & $\mathbf{V}$ & $\%$ & M & $\%$ & $\mathbf{V}$ & $\%$ & $\mathbf{M}$ & $\%$ \\
\hline \multirow{5}{*}{$\begin{array}{l}\text { Matemática } \\
\text { General }\end{array}$} & Ingeniería Industrial & 1 & 100 & - & - & 5 & - & 5 & - \\
\hline & Ingeniería en Sistemas (G1) & 1 & - & - & - & 2 & - & 4 & - \\
\hline & Ingeniería en Sistemas (G2) & 1 & - & - & - & 3 & - & 3 & - \\
\hline & Administración de Empresas & 1 & - & - & - & 2 & - & 3 & - \\
\hline & Psicología & 1 & - & - & - & 1 & - & 4 & - \\
\hline \multicolumn{2}{|r|}{ Sub total } & 5 & 100 & - & - & 13 & 41 & 19 & 59 \\
\hline \multirow{7}{*}{$\begin{array}{l}\text { Lengua y } \\
\text { Literatura }\end{array}$} & Ciencias Sociales & 1 & - & - & - & 1 & 13 & 7 & 87 \\
\hline & Pedagogía & 1 & - & - & - & 3 & 43 & 4 & 57 \\
\hline & Lengua y Lengua y Literatura & - & - & 1 & - & - & - & 2 & 100 \\
\hline & Mercadotecnia & 1 & - & - & - & 2 & 33 & 4 & 67 \\
\hline & Administración de Empresas & - & - & 1 & - & 3 & 43 & 4 & 57 \\
\hline & Inglés & - & - & 1 & - & 5 & 42 & 7 & 58 \\
\hline & Ciencias Naturales & - & - & 1 & - & 2 & 67 & 1 & 33 \\
\hline \multicolumn{2}{|r|}{ Sub total } & 3 & 43 & 4 & 57 & 16 & 35 & 29 & 65 \\
\hline TOTAL & & 10 & 57 & 7 & 43 & 38 & 30 & 81 & 70 \\
\hline
\end{tabular}

Es importante señalar, que durante el proceso de seguimiento, los alumnos mentores contaron con formatos de registro, seguimiento e informes para poder medir el avance del programa.

\section{RESULTADOS DEL PROCESO DE APLICACIÓN}

La ejecución del Programa Alumnos Mentores fue realizada durante el primer semestre 2016, encontrándose los siguientes logros y dificultades.

\subsection{Principales Logros}

\section{En relación a los alumnos mentores}

a. Estudiantes cualificados y motivados para ayudar a sus compañeros a lograr el éxito en una determinada asignatura.

b. Mejora en la interacción docente-estudiante, en cuanto a la necesidad de la planificación conjunta, entre ellos, de las sesiones de acompañamiento realizadas.

c. Mejora en la Práctica docente de los mentores de las carreras de Ciencias de la Educación.

d. Reconocimiento institucional, en la medida que se dieron a conocer frente a las autoridades universitarias y frente al estudiantado como alumnos mentores.

e. Entrega oportuna de material didáctico y gestión de los espacios donde se iba a ejercer la mentoría. 


\section{En relación a los estudiantes mentoriados}

a. Mejora en sus competencias académicas en relación a la asignatura en la que tenían dificultades.

b. Excelentes resultados académicos, ya que le permitió adquirir los aprendizajes de una forma más significativa al ser los mismos compañeros de la facultad quienes a su mismo nivel de estudiantes compartían sus conocimientos de forma desinteresada.

c. Los estudiantes aprenden mejor en un ambiente de amistad, afecto y estímulo.

\subsection{Dificultades encontradas}

a. Inasistencia de algunos alumnos mentoriados.

b. Choque de actividades planificadas con las sesiones de mentoría.

c. Incumplimiento de los compromisos adquiridos con los estudiantes mentores (no se otorgó la beca a todos los participantes mentores como fue prometido)

\section{REFERENCIAS}

Andrés, S. \& Barrios, A. (2006). El modelo del alumno ayudante a discusión: la opinión de los alumnos participantes y sus beneficiarios. Revista Electrónica de Investigación Psicoeducativa. No 9 Vol. 4 (2), 2006. ISSN: 1696-2095. pp. 311-nn. Extraído de: http://www.investigacionpsicopedagogica.org/revista/articulos/9/ espannol/Art_9_129.pdf

Espinar, S. R. (2015). Los estudiantes universitarios de hoy: una visión multinivel. Revista de Docencia Universitaria REDU, 91-124.

López, M. \& Sánchez, A. (2016). Programa de Estudiantes Mentores. UNAN-Managua, Vicerrectorado de Docencia.
Rey, Car. (1999). Alcanzando el Futuro: el papel de la mentoría en el nuevo milenio. Disponible en: www.mentors.ca

UNAN-Managua. (2011). Modelo Educativo Normativa y Metodologia para la Planificación Curricular 2011. Managua: UNAN-Managua.

Valverde Macías, A., García Jiménez, E. \& Romero Rodríguez, S. (2000). La función tutorial en la Universidad de Sevilla. La mentoría como respuesta: El proyecto S.I.M.U.S. 